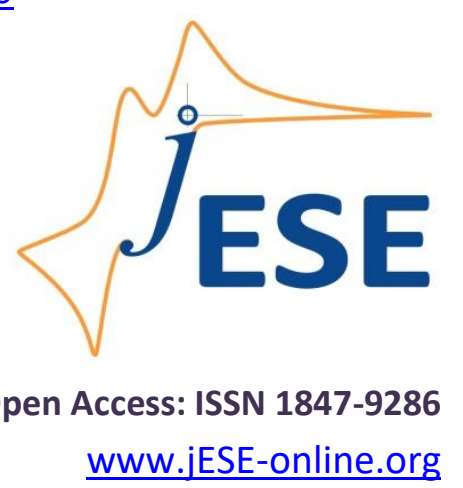

Original scientific paper

\title{
Principles of the express method for controlling interelectrode space condition during wire electrochemical processing
}

\author{
Vasyl Osypenko, Oleksandr Plakhotnyi ${ }^{\bowtie}$ and Oleksii Timchenko \\ Cherkasy State Technological University, Cherkasy, 18006, Ukraine \\ Corresponding author: ${ }^{凶}$ o.plakhotny@chdtu.edu.ua \\ Received: February 3, 2019; Revised: June 26, 2019; Accepted: June 27, 2019
}

\begin{abstract}
In the practical implementation of the sequential wire electrical discharge machining pulsed electrochemical machining (WEDM - PECM) technology and in order to perform high quality electrochemical processing, there is a need for the real-time operational control of electrical parameters of inter-electrode space and corresponding adaptive correction of amplitude-frequency power supply parameters (AFPSP). In the context presented by the authors, a mathematical apparatus and an algorithm of operational galvanostatic mode monitoring of anode dissolution using wire electrode-tool are proposed. This will allow adaptive adjustment of AFPSP to ensure controlled passage of electrochemical reactions and significantly increase process stability, dissolved surface layer thickness uniformity along entire electrode tool movement trajectory and resulting surface quality.
\end{abstract}

\section{Keywords}

Wire electrical discharge machining; pulsed electrochemical machining; current and voltage waveforms; process monitoring; surface finishing.

\section{Introduction}

Wire electrical discharge machining (WEDM) technology fully satisfies the requirements of modern production in terms of miniaturization and precision. However, modern industry and especially instrumental production require electrical discharge wire cutting machines to form surfaces with roughness $R a<0.1$ microns. Obtaining such surface parameters and getting rid of a structurally modified heat affected zone (HAZ) using only material removal by a highly concentrated source of heat having a spark discharge is extremely costly and difficult. In modern machines of the world leader in the field of electrical discharge nanotechnology, Sodick Co. LTD, a mirror-like smoothing of steel blank surfaces of up to 0.08 microns is achieved in 12 passes with a change of working environment. This leads to an unacceptable cost increase of machined parts. 
Pulse electrochemical machining (PECM) principally allows obtaining surfaces with roughness $R$ a $<0.1$ microns with the absence of a structurally altered layer on the workpiece surface. However, implementation of an efficient PECM process by a mobile wire tool electrode (WTE) following to the WEDM technology scheme is currently not sufficiently researched and is challenging in scientific and technical terms. The solution requires complex experimental and theoretical studies, as well as mathematical and physical modelling of basic processes that determine the nature of the electrochemical dissolution of the anode surface obtained by WEDM.

\section{Literature review and problem statement}

For WEDM, the express methods of preliminary determination of power supply parameters and inter-electrode space (IES) condition monitoring in real time are already developed and successfully used on serial machines. The IES resistance is controlled by the hardware. AFPSP and machine drives feed rate have adaptive control. More sophisticated modern schemes are implemented through the continuous supply of low-power diagnostic impulses to IES. By analysing the response, a decision is made to switch on a more powerful source of pulsed technological current, which in fact, carries the electro-discharge destruction of material [1,2].

For wire PECM, such control methods have not been developed. The time of periodic process of double layer charging to the overvoltage activation and its discharge time are very important for nanosecond pulsed electrochemical processing [3-5]. Cylindrical wire electrode causes significant current density distribution on the anode surface. Therefore, even a slight change in electrode potential leads to a significant change in current density and accordingly, to dissolution localization. With a longer duration of microsecond pulses, a diffusion processes becomes a limiting factor in IES. Accordingly, calculation of stationary and transition components of diffusion current, which depend on main parameters of each technological PECM scheme, must be preceded by the choice of current amplitude [6]. For configuration of flat anode and cylindrical cathode with stream electrolyte flow, it is problematic to determine diffusion layer thickness, which depends on velocities distribution of electrolyte in near-anode zone. Consequently, calculated mathematical models of electrochemical processes in IES are complex, require usage of computer-aided design software and significant computational time [7]. These mathematical models are adequate for preliminary calculation of PECM processes parameters but are not suitable for operational monitoring and adaptive real-time correction. Therefore, there is an urgent need for the creation of algorithms and schemes suitable for practical implementation in the existing technological equipment.

The proposed approach is based on the determination of magnitude and dynamics change of IES electrical parameters and as a result, process nature of electrochemical dissolution. These can be obtained from analysis of response oscillograms using equivalent electrical circuit substitution scheme. A similar approach was used in [8], using a complex mathematical apparatus not suitable for operative quantitative analysis of response oscillograms. In the subsequent work [9] only a qualitative analysis of oscillograms was conducted.

Proper choice of AFPSP according to PECM scheme, can significantly improve surface quality, machining accuracy, material removal rate and overall process controllability $[10,11]$.

The purpose is to increase the technological characteristics of high-performance combined methods of current-conducting materials machining with the help of further development of mathematical apparatus of operational monitoring and adaptive efficiency correction of galvanostatic PECM mode. 


\section{Experimental equipment and methods}

\section{Technological scheme}

When using the PECM scheme of unipolar current pulses with moving WTE as a cathode (Figure 1), developed by the authors and implemented in particular experimental technological equipment, the process of anodic dissolution of workpiece material significantly depends on the magnitude and growth dynamics of anode polarization and relaxation of its potential during the pause between current pulses.

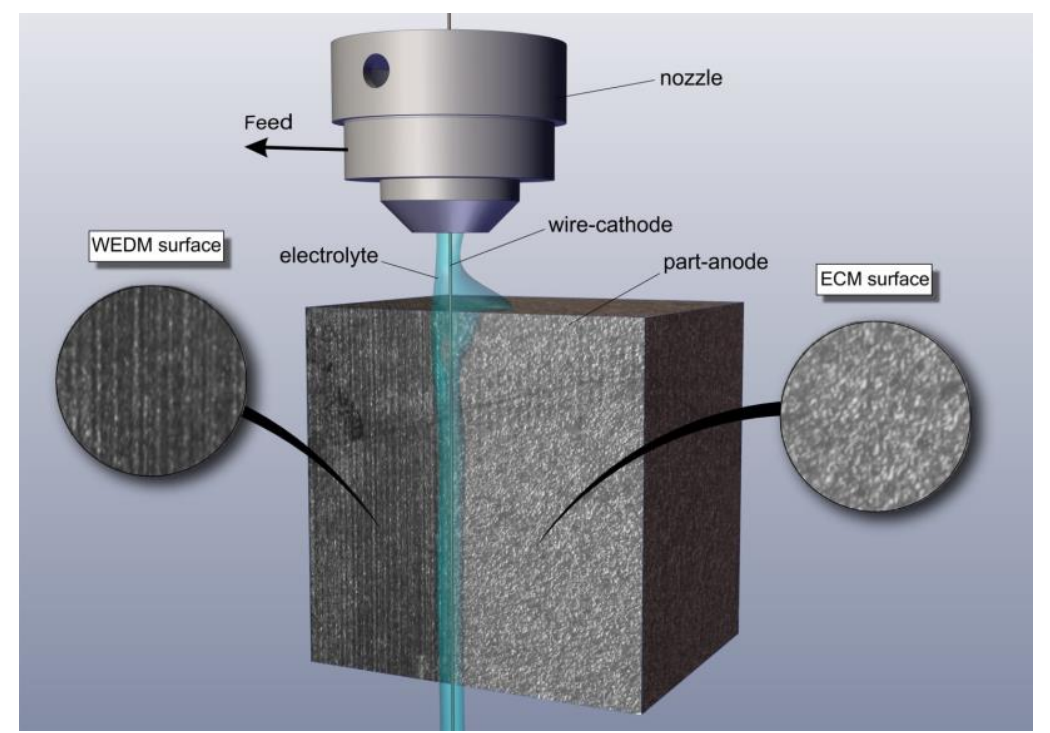

Figure 1. Machining scheme of sequential wire EDM and wire PECM

Replacement of the partition boundary "anode - electrolyte" for equivalent electrical circuit (Randles circuit) is carried out and described in Figure $2[8,12]$. The choice of circuit should be based on electrical properties of IES. Technological parameters during machining process determine dynamics change of electrical properties of IES. Electrolyte flow speed (renewal rate) influences $R_{E}$ - electrolyte resistance. Change of IEG value, a size of interacting surface while moving on intricatecontoured trajectory affects capacity. WTE feed rate along trajectory alters dissolution conditions of EDM-affected surface layers and influences the electrical properties of IES.

a

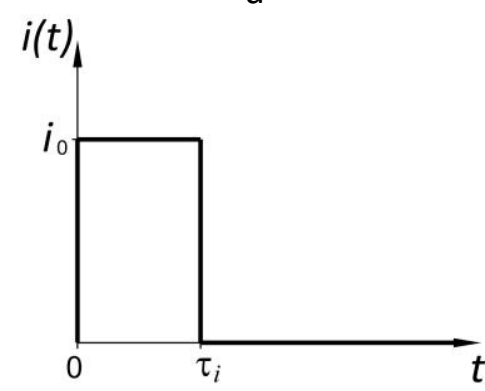

b

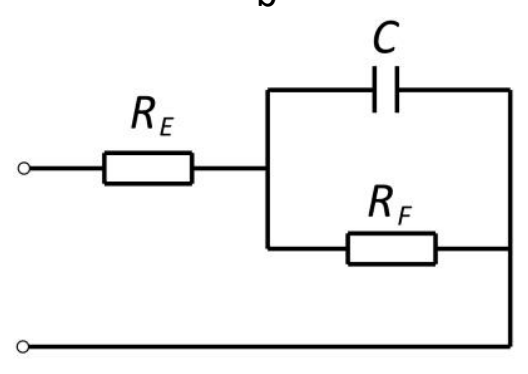

Figure 2. $\boldsymbol{a}$-Current pulse parameters; $\boldsymbol{b}$ - Electrical equivalent scheme for anode-electrolyte boundary interface of pulse ECM (PECM): $R_{E}$ - electrolyte resistance; $C$-double layer capacity;

\section{$R_{F}$ - Faraday resistance}

Methods for obtaining oscillograms of anode current pulses are well worked out and have a relatively simple scheme implementation (Figure 3) $[8,13]$.

Research of physical and technological parameters of wire cutting electrical discharge machining and subsequent electrochemical processing by a wire electrode was carried out on SELD-02 (wire-cut 
electrical discharge machine on linear motors, Ukraine). SELD-02 machine has linear motors, granite guides and gas-lubricated supports. This provides minimal displacement error.

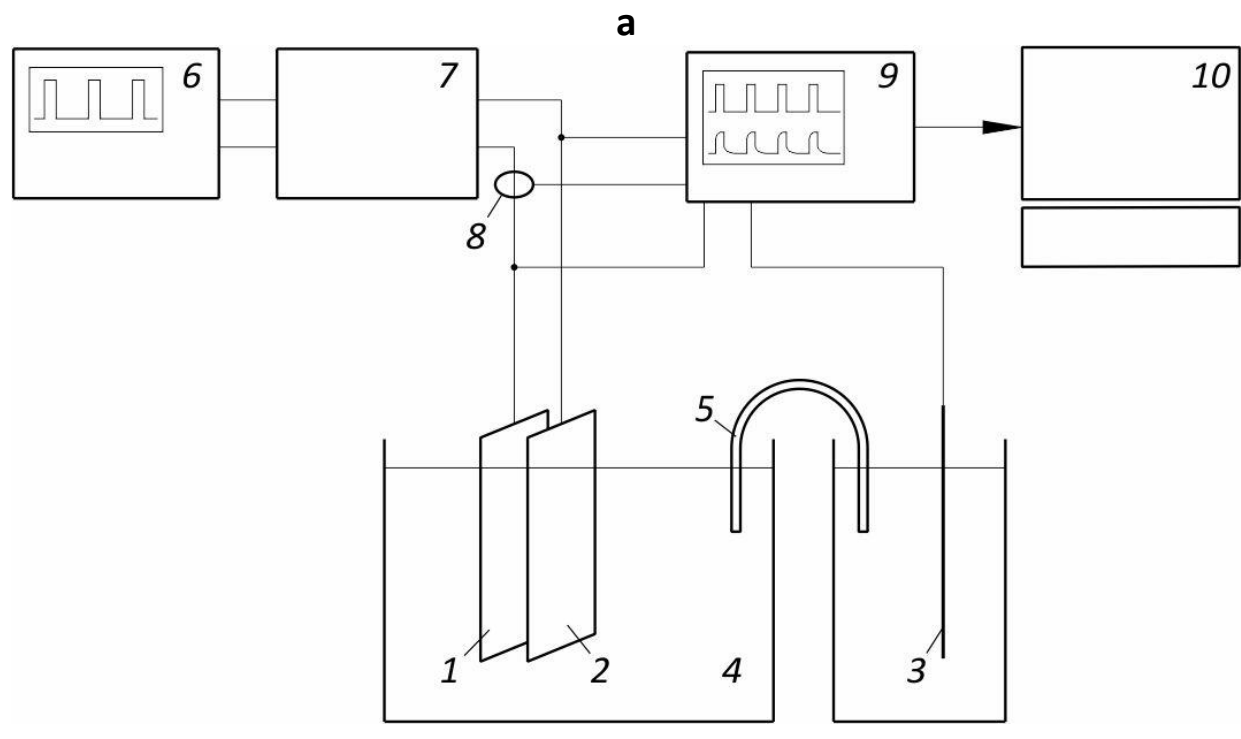

b

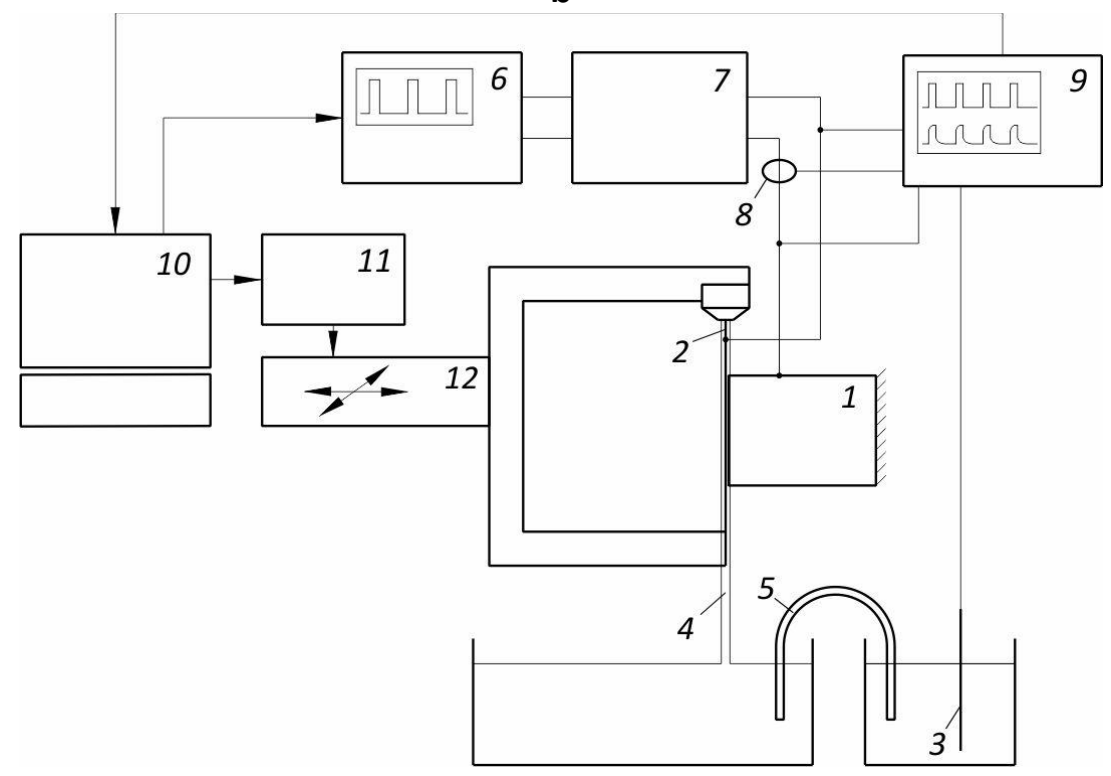

Figure 3. Schemes of measurements: $\boldsymbol{a}$-flat electrodes, $\boldsymbol{b}$ - flat anode -cylindrical (wire) cathode. 1 -anode, 2 -cathode, 3 -reference electrode (platinum), 4 -electrolyte, 5 -bridge, 6 -driving pulse generator, 7 -current source, 8 -current transducer, 9 -oscillograph, 10 computer, software, 11 - computer numerical control unit, 12 - positioner

Experimental conditions: anode - stamping steel DIN X155CrVMo12-1, cathode $-0.2 \mathrm{~mm}$ diameter Cobra Cut B wire (AGIE, Switzerland), CuZn37 hard brass, $1 \mathrm{M} \mathrm{NaCl}$ electrolyte at temperature of $28{ }^{\circ} \mathrm{C}$ which was delivered as a jet from the upper chamber of the machine at pressure of $5 \times 10^{4} \mathrm{~Pa}$. Height of the part is $11 \mathrm{~mm}$. The machine-tool provided wire electrode movement with a given speed along the trajectory at a given distance (IEG) from the machined surface. Conductivity of the electrolyte was controlled by the Oakton TDS-5/CON-5 with a special electrode which expands measurement scale (cell factor $K=10$ ). Polarization-time and current-time dependencies were recorded by digital Atten ADS1000 dual-channel oscillograph and transmitted to computer in a mathematical software package for processing.

Preliminary testing of the proposed method for monitoring IES was carried out in studies of transient processes in electrochemical system. It had fixed flat electrodes and the process was 
conducted by the passing of a sequence of rectangular current pulses. Electrodes $20 \times 12.5 \times 1.5 \mathrm{~mm}$ were immersed in a bath with immovable $1 \mathrm{M} \mathrm{NaCl}$ electrolyte, so the electrodes interaction area was $2.5 \mathrm{~cm}^{2}$. Anode material - stamping steel DIN X155CrVMo12-1, cathode - brass CuZn37.

Mathematical method for calculating parameters of an electric circuit loaded by microsecond current pulses

As is known, current $i(t)$ and voltage $u(t)$ at the ends of an electrical circuit element with active resistance $R$ and capacity $C$ are bound by formula [14]

$$
u(t)=R \cdot i(t), \quad u(t)=\frac{1}{C}\left[\int_{0}^{1} i(t) \mathrm{d} t+q_{0}\right]
$$

where $q_{0}$ - initial charge on condenser covers.

Using the operational method [14], we introduce an integral transform of Laplace's "operational current", $i(t) \rightarrow I(p)$ and "operational voltage", $u(t) \rightarrow U(p)$, where $p$ is a complex number frequency parameter. Then, relation (1) will be converted into operational formulas

$$
U=R I, \quad U=\frac{1}{C p} I
$$

if we consider $q_{0}=0$.

Formulas (2) combine into "operational Ohm's law "

$U=Z I$

where $Z$ is "operational resistance" or impedance, which in the case of active resistance and capacitance has the following form:

$$
Z_{R}=R, \quad Z_{C}=\frac{1}{C p}
$$

Considering rules for adding parallel and serial impedances, we obtain the expression for the overall circuit (Figure $2 b$ ) impedance,

$$
Z=R_{E}+\frac{R_{F}}{C p R_{F}+1}
$$

and the operational equation, respectively:

$$
U=\left(R_{E}+\frac{R_{F}}{C p R_{F}+1}\right) I
$$

Similarly, it is possible to formulate the operational equation for more complex electric substitution schemes taking into account additional components of inter-electrode space, such as cathode double electrical layer capacitance, resistance of passivating films that can be formed on surface of electrodes, etc. Also, in the substitution scheme, inductivities can be introduced if response oscillograms taken from IES clearly indicate oscillatory attenuation signals and model must reproduce them. It is important to substantiate the conformity of each element of electrical circuit and way of its connection to the real processes occurring in IES during electrochemical machining.

To construct a mathematical description of the passage of a rectangular current pulse through a model of an electrochemical cell (Figure $2 \mathrm{~b}$ ), we first consider the problem of DC current $i_{0}$ activation at time $t=0$. 
$i(t)=i_{0} \theta(t), \quad \theta(t)=\left\{\begin{array}{l}0, \text { if } t<0, \\ 1, \text { if } t \geq 0 ;\end{array}\right.$

$\theta(t)$ - Heaviside function.

Operational current is defined as $I=i_{0} / p$, while the operational voltage according to Eqs. (3) and (6) is defined as:

$$
U=\left(R_{E}+\frac{R_{F}}{C p R_{F}+1}\right) \frac{i_{0}}{p}
$$

We perform the transition operation to the original $u(t) \leftarrow U(p)$ started using the conclusion of the second expansion theorem [14], according to formula

$$
\frac{A(p)}{p B(p)} \rightarrow \frac{A(0)}{B(0)}+\sum_{k=1}^{k=1} \frac{A\left(p_{k}\right)}{p_{k} B^{\prime}\left(p_{k}\right)} e^{p_{k} t}
$$

where $p_{\mathrm{k}}-\mathrm{k}^{\text {th }}$ equation root of $B(p)=0$.

In our case, using Eq. (7) we have only one root, $p_{1}=1 / C R_{F}$. Then using Eq. (8)

$$
\frac{A(p)}{p B(p)} \rightarrow\left(R_{E}+R_{F}\right) i_{0}-R_{F} i_{0} e^{p_{1} t}
$$

$u(t)$ becomes defined as:

$$
u(t)=i_{0}\left[R_{E}+R_{F}\left(1-e^{p_{1} t}\right)\right]
$$

Next, to get response function to the rectangular shape current pulse passage, $i(t)=i_{0} \theta(t)-i_{0} \theta\left(t-\tau_{i}\right)$, where $\tau_{i}$ is pulse duration, the lag theorem [14] was used and we get:

$$
u(t)=i_{0}\left\{\left[R_{E}+R_{F}\left(1-e^{p_{1} t}\right)\right] \theta(t)-\left[R_{E}+R_{F}\left(1-e^{p_{1}\left(t-\tau_{i}\right)}\right)\right] \theta\left(t-\tau_{i}\right)\right\}
$$

Here it is assumed that electrochemical cell parameters $R_{E}, R_{F}, C$ have not changed during pulse duration.

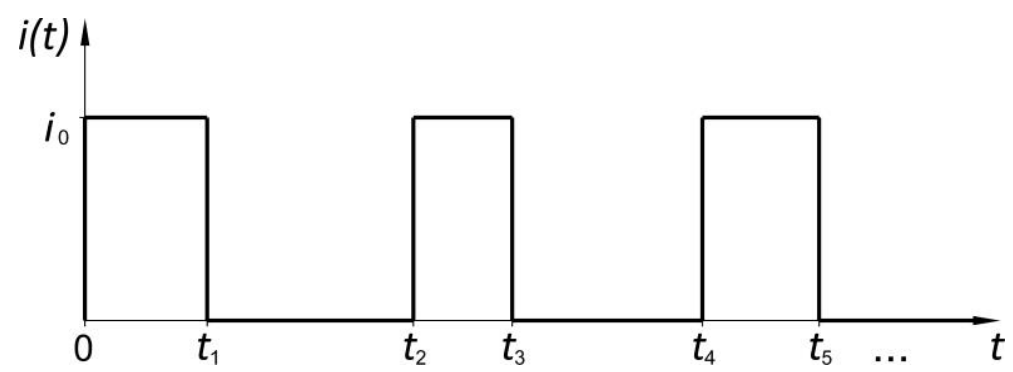

Figure 4. Sequence of current pulses with arbitrary parameters of duration and on-off time

For a series of current pulses with an arbitrary time duration and on-off time parameters (Figure $4)$, it is suitable to use the following form of the response function

$$
\begin{aligned}
& u(t)=i_{0}\left\{\left[R_{E 1}+R_{F 1}\left(1-e^{p_{1} t}\right)\right] \theta(t)-\left[R_{E 2}+R_{F 2}\left(1-e^{p_{2}\left(t-t_{1}\right)}\right)\right] \theta\left(t-t_{1}\right)+\right. \\
& +\left[R_{E 3}+R_{F 3}\left(1-e^{p_{3}\left(t-t_{2}\right)}\right)\right] \theta\left(t-t_{2}\right)-\left[R_{E 4}+R_{F 4}\left(1-e^{p_{4}\left(t-t_{3}\right)}\right)\right] \theta\left(t-t_{3}\right)+ \\
& +\left[R_{E 5}+R_{F 5}\left(1-e^{p_{5}\left(t-t_{4}\right)}\right)\right] \theta\left(t-t_{4}\right)-\left[R_{E 6}+R_{F 6}\left(1-e^{p_{6}\left(t-t_{5}\right)}\right)\right] \theta\left(t-t_{5}\right)+ \\
& +\ldots\},
\end{aligned}
$$

where according to the trend of parameters changing from $R_{E 1}, R_{F 1}, C_{1}$ to $R_{E 2}, R_{F 2}, C_{2}$, then to $R_{E 3}, R_{F 3}$, $C_{3}$, etc, it is possible to clearly observe dynamics of IES condition indicators for an electrochemical cell during pulsed current workload in the galvanostatic mode. 


\section{Results and discussion}

\section{Flat electrodes}

Figure 5(a) presents the results of dynamic measurements of anode polarization at given amplitude-time parameters of current. It should be noted that amplitude of current pulses and interaction area of electrodes in this experiment do not correspond to the operating modes of current density of electrochemical dissolution using wire WEDM + PECM technology. Duration and amplitude of pulse current, area of interaction of electrodes, IEG value were selected in such a way to maximize manifestation of IES resistance and capacity in anodic polarization curves. In addition, oscillograms were recorded at the beginning of dissolution process in order to minimize influence of external factors such as electrochemical reaction products saturation (slag) and IES gas pollution or formation of various films on electrodes, which had not yet appeared in a short time.

a

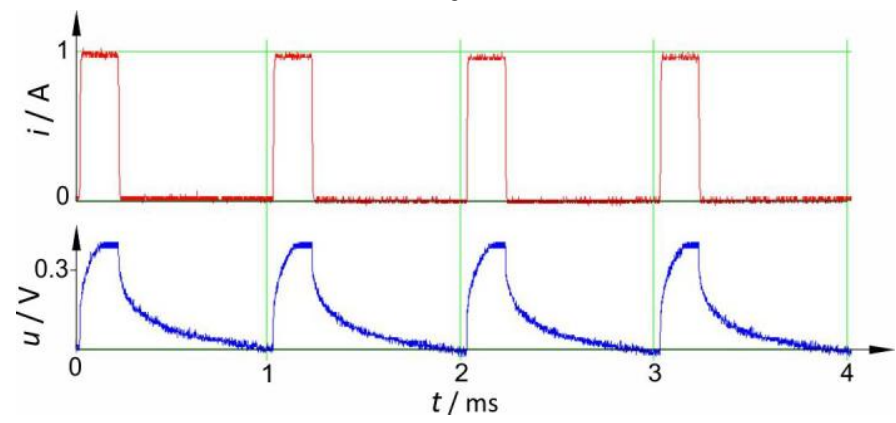

b

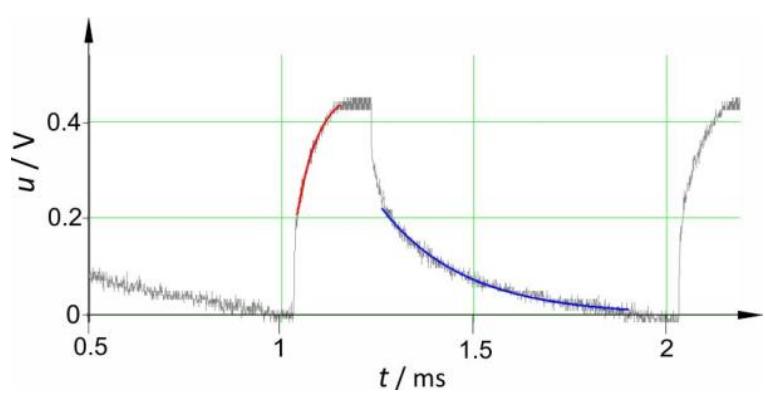

Figure 5. Oscillograms and their mathematical processing: $\boldsymbol{a}$ - rectangular current pulses (red curve); increase and decrease of anode polarization (blue curve); $\boldsymbol{b}$ - approximation by response function of areas with transient processes

Then, the inverse mathematical problem was solved using the experimentally obtained plot of polarization-time dependence, and selecting coefficients in the response function (Eq. (10)), i.e. parameters $R_{\mathrm{E}}, R_{\mathrm{F}}, C$ of the electrical equivalent substitution scheme of an electrochemical cell. Polarization growth area (Figure $5 b$ ) was approximated by the least squares method using function (9), which is a response function when passing the front edge of current pulse through an electrochemical cell. The decrease of area which corresponds to the back edge of the switch-off of current pulse, was approximated by the function represented in the second term Eq. (10).

According to oscillogram processing results (Figure $5 b$ ), it was found that at the moment of passing the forward front of current pulse, IES parameters had following values: $R_{\mathrm{E}}=0.12 \mathrm{Ohm}$, $R_{\mathrm{F}}=0.33 \mathrm{Ohm}, C=1.2 \cdot 10^{-4} \mathrm{~F}$. At the moment of passing rear front, these values were: $R_{\mathrm{E}}=0.12 \mathrm{Ohm}$, $R_{\mathrm{F}}=0.4 \mathrm{Ohm}, C=3.9 \cdot 10^{-4} \mathrm{~F}$. Estimated total IES resistance, $R$, according to the Ohm's law

$$
R=\frac{\delta}{\kappa S}
$$

using values of electrolyte conductivity, $\kappa=8.61 \mathrm{~S} \mathrm{~m}^{-1}$, electrodes interaction area, $S=2.5 \cdot 10^{-4} \mathrm{~m}^{2}$ and IEG, $\delta=0.8 \cdot 10^{-3} \mathrm{~m}$, was $0.37 \mathrm{Ohm}$. Specific capacity of double electric layer i.e. capacity according to the equivalent interaction area of electrodes is $0.48 \mathrm{~F} / \mathrm{m}^{2}$ and $1.6 \mathrm{~F} / \mathrm{m}^{2}$ in the first and second case, respectively. According to the literature data, specific capacity values should be within 0.2-0.4 F/m ${ }^{2}$ [15], Helmholtz model, 0.6-0.8 F/m ] [15], or 0.4-0.5 F/ $\mathrm{m}^{2}$ [16], measured at the boundary between mercury anode and $1 \mathrm{M} \mathrm{NaCl}$ solution at $25^{\circ} \mathrm{C}, 0<\mathrm{U}<0.4 \mathrm{~V}$. That is, the order of experimentally determined resistance values $R_{\mathrm{E}}+R_{\mathrm{F}}$ (values are adding for serial connection) 
coincides with theoretical calculation. The found specific capacity value along the pulse forward front is located in well-known range. The rear edge determined capacity, however, is not correct (similar to [8]), so it is obvious that the electrical equivalent scheme needs to be clarified.

With an increase of the current amplitude up to $9 \mathrm{~A}$ and a decrease in of IEG value down to $0.6 \mathrm{~mm}$, IES is filled with sludge over time due to reactions products. This is noticeable when analysing oscillograms (Figure 6). At the beginning of experiment, electrochemical cell specific capacities are $0.22 \mathrm{~F} / \mathrm{m}^{2}$ and $0.76 \mathrm{~F} / \mathrm{m}^{2}$ for forward and rear pulse fronts, respectively. After 2 minutes of the anode dissolution, a significant amount of sludge is observed visually in IES, and from analysing oscillograms it follows that the corresponding capacities increased to $0.49 \mathrm{~F} / \mathrm{m}^{2}$ and $1.2 \mathrm{~F} / \mathrm{m}^{2}$ and resistance decreased. Consequently, IES is filled by sludge, caused by insufficient rate of stationary electrolyte renewal that is clearly visible on the oscillograms and can be quantified by their mathematical analysis.

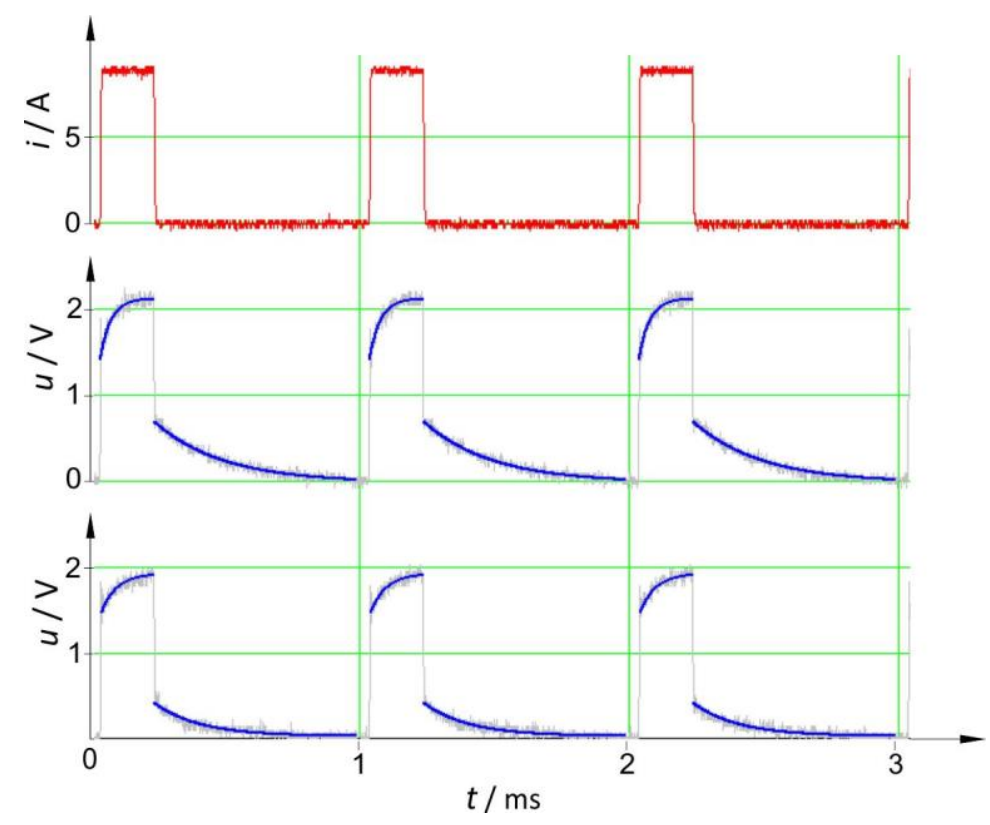

Figure 6. Oscillograms, approximated by response function, when working with a larger current amplitude 9A: voltage at the beginning of experiment-medium graph; voltage after 2 minutes of polluted IES - lower graph

With a variation of the IEG and AFPSP parameters, other characteristic cases of polarization change dynamics of electrodes were experimentally obtained. Oscillograms in Figure 7 are mathematically processed in the following way. On the first pulse, we obtain resistance and capacitance parameters of elements and using Eq. (11) further polarization change is predicted for several subsequent pulses (red curve).

In the first case (Figure 7a), an unsuccessful choice of AFPSP for experimental conditions led to increasing residual polarization. In another example (Figure $7 \mathrm{~b}$ ) there is an increase in polarization and a simultaneous change in IES conditions. Both cases are unacceptable and require an immediate correction of AFPSP for normalization of the anode dissolution process in electrochemical cell with flat electrodes. A novel pulsed-power supply method allows to accelerate depolarization and to improve the machining accuracy of PECM [17]. 
a
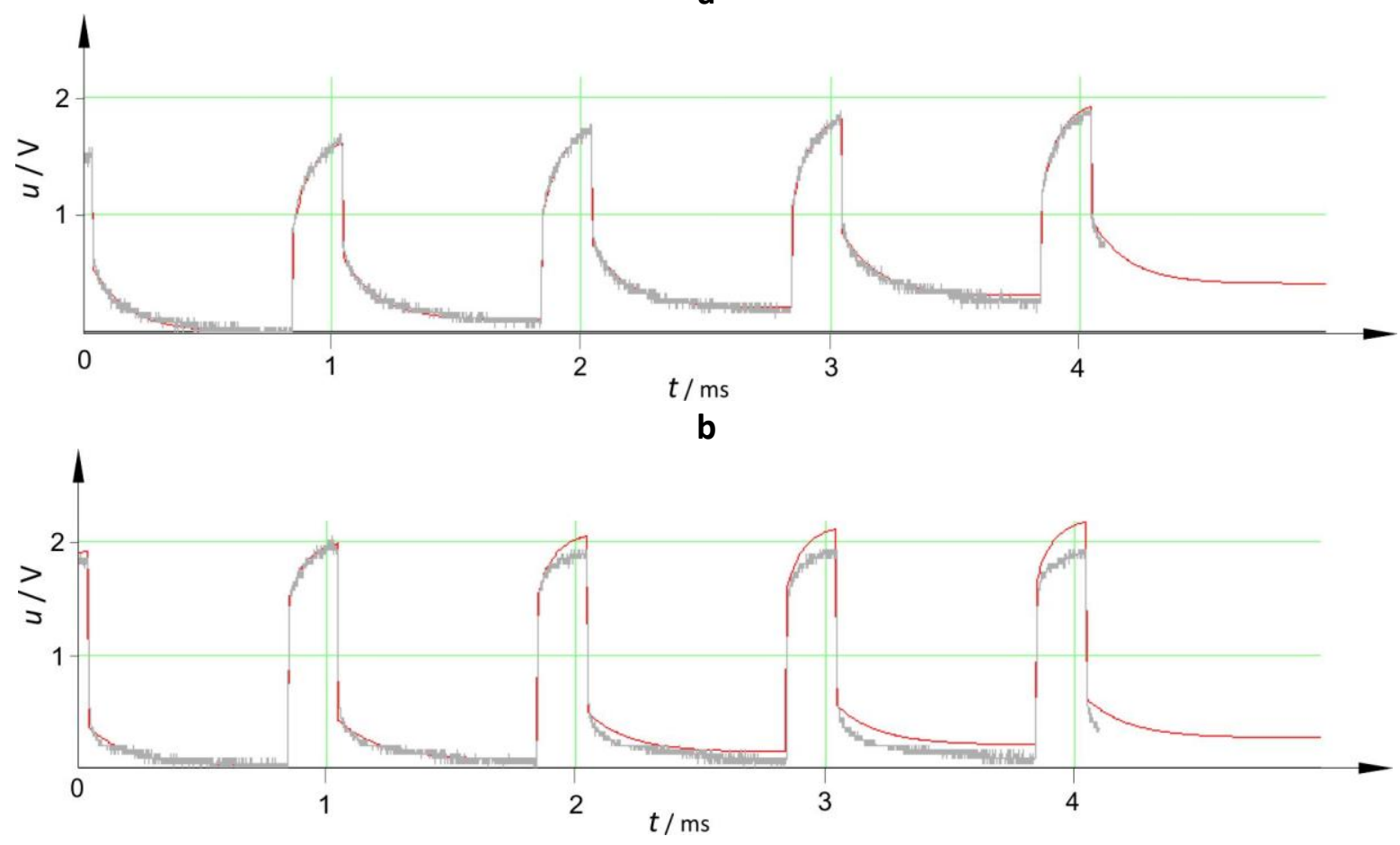

Figure 7. Oscillograms with (a) increasing residual polarization, (b) increasing polarization and changes in IEG characteristics

Flat anode - cylindrical cathode

For polarization oscillograms with flat anode and wire cathode analysis it is incorrect to use value of electrodes interaction area $S$ in Eq. (12). To obtain more accurate quantitative results of oscillograms analysis, the following mathematical description of electric field distribution for the calculation scheme of flat anode - cylindrical cathode (Figure 8) $[18,19]$ must be applied.

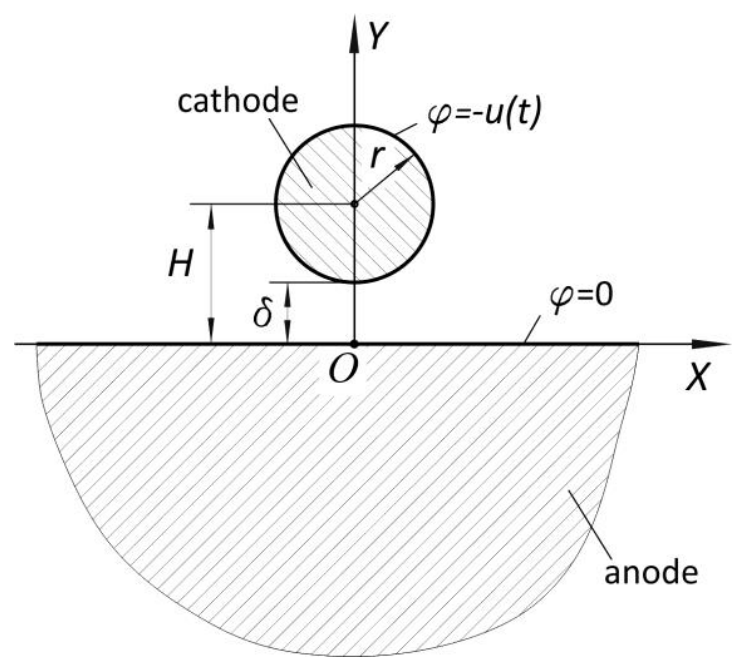

Figure 8. Scheme for electrochemical machining of flat anode by a wire cathode

If cathode potential is $u(t)$, then field strength distribution on anode surface is determined by the formula

$$
\left.E_{y}(x, t)\right|_{y=0}=\frac{2 a u(t)}{\ln p} \frac{1}{x^{2}+a^{2}}
$$

where $a=\sqrt{H^{2}-r^{2}}, p=(a-\delta) /(a+\delta)$. Field strength component $E_{x}$ is insignificant due to smallness. 
Amplitude current in circuit can be described by the following equation

$$
I_{\mathrm{A}}=\left.2 \int_{0}^{\infty} \kappa E_{y}\right|_{y=0} L \mathrm{~d} x=2 \pi L \kappa \frac{U_{\mathrm{A}}}{|\ln p|}
$$

where $\kappa$ is specific electrolyte conductivity, and $L$ is the part height.

Then IES resistance filled with electrolyte is

$$
R=\frac{|\ln p|}{2 \pi L \kappa}
$$

or by analogy with Eq. (12)

$$
R=\frac{a}{\kappa A_{\mathrm{s}}}
$$

where, $A_{\mathrm{s}}=2 \pi a L /|\ln p|$ i.e. equivalent interaction area for cylindrical cathode and flat anode, similar to the interaction area of electrodes $S$ for flat electrodes.

The area of electrodes interaction, which in turn determines electrical capacity of electrochemical cell, has much less calculated value for a wire electrode than for a pair of flat electrodes. Therefore, transient processes of increasing and decreasing polarization under pulsed current are visually less obvious. Figure 9 depicts an oscillogram of anode polarization at IEG of $0.5 \mathrm{~mm}$, amplitude current $I_{\mathrm{A}}=2.34 \mathrm{~A}$ and voltage $U_{\mathrm{A}}=11 \mathrm{~V}$.

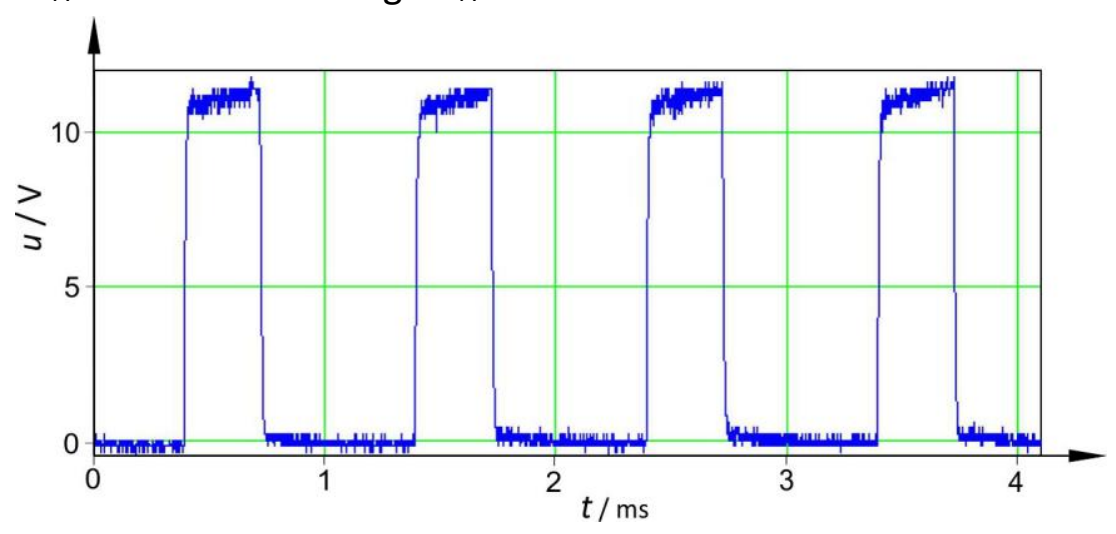

Figure 9. Oscillogram of anode polarization during electrochemical machining of flat anode by a wire cathode.

Figure 10 shows areas of voltage growth and decrease of oscillogram in Figure 9, approximated by the response function (Eq. 10). The experimental value of resistance is $4.7 \mathrm{Ohm}$, while the theoretically calculated value according to Eq. (15) is $4.33 \mathrm{Ohm}$. The difference between the experimental and theoretical results is $7.9 \%$ and may be explained by the appearance of partial gas pollution of IES as a result of electrochemical reaction, which leads to a decrease in electrolyte conductivity.

Following the results of the response function approximation of forward pulse front, the following values were obtained: $R_{\mathrm{E}}=3.74 \mathrm{Ohm}, R_{\mathrm{F}}=0.89 \mathrm{Ohm}, C=8.3 \cdot 10^{-6} \mathrm{~F}$. Specific capacity, i.e. the capacity of electrochemical cell according to the equivalent interaction area of electrodes, is $0.5 \mathrm{~F} / \mathrm{m}^{2}$. Data of the voltage drop area approximation asserts that specific capacity reached $0.45 \mathrm{~F} / \mathrm{m}^{2}$.

With the prolongation of response function according to Eq. (11) with parameters obtained for several subsequent pulses and comparison with the oscillogram in Figure 9, it was established that electrochemical process proceeds normally, without significant changes in IES condition and without accumulation of residual polarization. That is, AFPSP for given conditions is selected correctly, providing necessary metal removal rate and avoiding a process of entering passivation zone. 
a

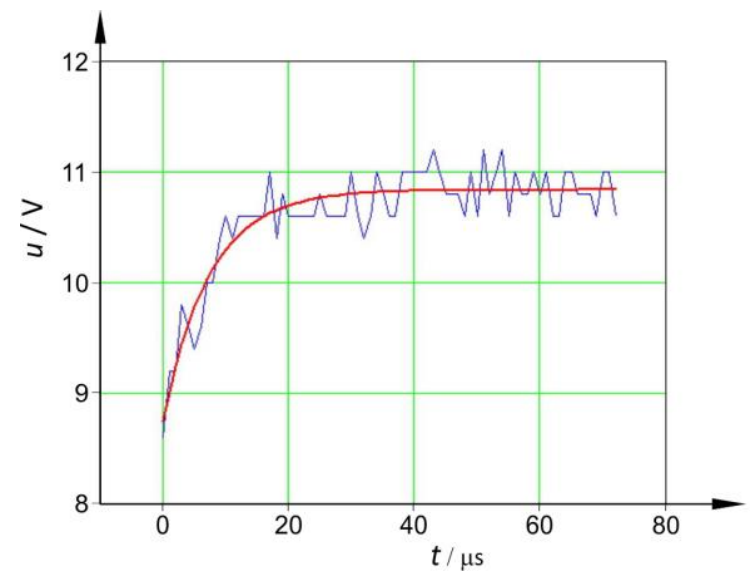

b

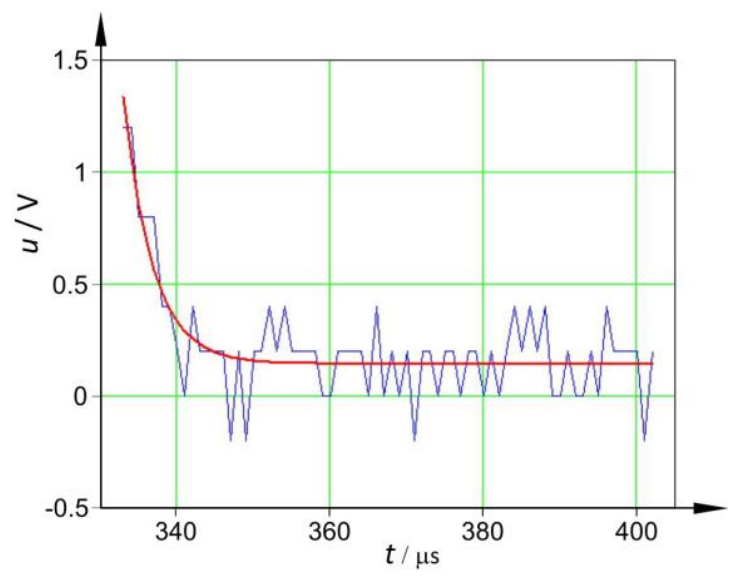

Figure 10. Voltage oscillogram (blue curve) approximated by response function (red curve) (a)-area of voltage increase on forward pulse front; (b) area of voltage decrease on rear pulse front.

Following the results of the response function approximation of forward pulse front, the following values were obtained: $R_{\mathrm{E}}=3.74 \mathrm{Ohm}, R_{\mathrm{F}}=0.89 \mathrm{Ohm}, C=8.3 \cdot 10^{-6} \mathrm{~F}$. Specific capacity, i.e. the capacity of electrochemical cell according to the equivalent interaction area of electrodes, is $0.5 \mathrm{~F} / \mathrm{m}^{2}$. Data of the voltage drop area approximation asserts that specific capacity reached $0.45 \mathrm{~F} / \mathrm{m}^{2}$.

With the prolongation of response function according to Eq. (11) with parameters obtained for several subsequent pulses and comparison with the oscillogram in Figure 9, it was established that electrochemical process proceeds normally, without significant changes in IES condition and without accumulation of residual polarization. That is, AFPSP for given conditions is selected correctly, providing necessary metal removal rate and avoiding a process of entering passivation zone.

According to Faraday's law we obtain an expression for determining material depth dissolution distribution on anode surface due to the action of one current pulse

$$
h(x)=\frac{2 K_{v} \kappa a \eta}{\ln p} \frac{1}{x^{2}+a^{2}} \int_{0}^{T} u(t) d t
$$

where $K_{\mathrm{v}}$ is coefficient of electrochemical machinability of anode material; $\eta$ is current efficiency coefficient; $T$ is pulse period; $u(t)$ is response function (10) obtained by approximating the corresponding voltage oscillogram section during current pulse passage.

If the process of electrochemical dissolution is stable as in the considered example, then according to Eq. (17) it is possible to calculate the thickness of the removed surface layer, taking into account the velocity of the wire electrode and current pulse frequency. In the case of ECM parameters deviation from normal reaction, oscillogram analysis will indicate unacceptable changes. Refusal to apply corresponding operational measures for correction of AFPSP will result in a significant reduction of current efficiency coefficient. This is approved by numerous experimental evidences in practical implementation of wire ECM technology.

Approximation of oscillogram signals in this work was carried out using a mathematical software package, which is quite convenient for research, but is not fundamentally necessary. Computational experiments have shown that sufficient accuracy in this case can provide an approximation of 3-5 points of the curve (filtered from digital noise). According to the least squares deviation method, this means that in order to find unknown coefficients, it is necessary to solve a system of 3-5 linear algebraic equations, which solution can be represented in an analytical form. That is, calculations are reduced to normal multiplication and addition operations that can be performed in real time on 
microprocessor elements used in modern computer numerical control equipment for controlling and managing machine-tools and technological systems.

\section{Conclusions}

1. The concept of operational monitoring, forecasting and adaptive correction of AFPSP of ECM using mobile wire electrode is substantiated in order to improve accuracy and quality of surface shaping using the latest combined technology of sequential WEDM and ECM.

2. Based on experimental data, a mathematical model was developed that adequately describes the increase and decrease of polarization dynamics of anode in electrochemical machining using wire electrode, with a series of unipolar rectangular current pulses.

3. The solution of inverse mathematical problem is proposed which, based on the experimentally obtained plot of the response polarization-time dependence, allows-determination of $R_{E}, R_{F}, C$ parameters of the substituted electrical equivalent circuit scheme of an electrochemical cell.

\section{References}

[1] V. I. Osypenko, S. P. Poliakov, D. O. Stupak, R. I. Savisko UA 10153 MPK 7B23H1/02 (2005).

[2] Y. Jiang, W. Zhao, X. Xi, L. Gu, X. Kang, International Journal of Advanced Manufacturing Technology 61(1-4) (2012) 171-183.

[3] R. Schuster, V. Kirchner, P. Allongue, G. Ertl, Science 289(5476) (2000) 98-101.

[4] S. Skoczypiec, A. Ruszaj, Precision Engineering 38(3) (2014) 680-690.

[5] S. Skoczypiec, International Journal of Advanced Manufacturing Technology 87(1-4) (2016) 177-187.

[6] C. Zhao, L. Xu, Journal of Electrochemical Science and Engineering 8(4) (2018) 321-330.

[7] J. A. Kenney, G. S. Hwang, Nanotechnology 16(7) (2005) S309.

[8] S. Galanin, Elektrokhimicheskaya obrabotka metallov i splavov mikrosekundnymi impul'sami toka (Electrochemical Treatment of Metals and Alloys by Microsecond Current Pulses), Kostrom. Gos. Tekh. Univ., Kostroma, Russia, 2001, p.118 (in Russian).

[9] S. Galanin, I. Kalinnikov, Surface Engineering and Applied Electrochemistry 44(5) (2008) 359-366.

[10] M. Mithu, G. Fantoni, J. Ciampi, International Journal of Advanced Manufacturing Technology 55(912) (2011) 921-933.

[11] G. Wollenberg, H. Schulze, H. Trautmann, G. Kappmeyer, Proceeedings of the $15^{\text {th }}$ ISEM, (2007) 335338.

[12] O. Weber, A. Rebschlager, P. Steuer, D. Bahre, Proceedings of the European COMSOL conference, Rotterdam, 2013.

[13] J. Sun, E. Taylor, R. Srinivasan, Journal of Materials Processing Technology 108(3) (2001) 356-368.

[14] M. A. Lavrent'ev, B. V. Shabat, Methods of the theory of function of complex variable, Nauka, Moscow, 1987, p.544 (in Russian).

[15] V. S. Bagotsky, Fundamentals of Electrochemistry. John Wiley \& Sons, Inc.: Hoboken, NJ, 2005, p.752.

[16] J. Newman, K.E. Thomas-Alyea, Electrochemical Systems, John Wiley \& Sons, NY, 2012, p.672.

[17] F. Han, W. Chen, W. Ying, Procedia CIRP 68 (2018) 493-498.

[18] V.P. Zhitnikov, A. N. Zaytsev, Impul'snaya elektrokhimicheskaya razmernaya obrabotka: monografiya (Pulse electrochemical machining: monograph), Mashynostroenye, Moscow, 2008, p. 413 (in Russian).

[19] V.I. Osipenko, A. P. Plakhotny, A. Yu. Denisenko, Pratsi Odeskoho politekhnichnoho universytetu 1(43) (2014) 55-60.

C2019 by the authors; licensee IAPC, Zagreb, Croatia. This article is an open-access article distributed under the terms and conditions of the Creative Commons Attribution license (http://creativecommons. org/licenses/by/4.0/) 